\title{
Messung kleiner Atomkonzentrationen in der Gasphase mittels Resonanzfluoreszenz im Vakuum-UV
}

\author{
K. H. Becker, W. Groth und W. Jud \\ Institut für Physikalische Chemie der Universität Bonn \\ (Z. Naturforsch. 24 a, 1953-1958 [1969] ; eingegangen am 7. Oktober 1969)
}

\begin{abstract}
The resonance fluorescence of atomic oxygen, nitrogen, krypton, and argon was measured in the vacuum-uv region as a function of the atom concentration. In a model the calculated fluorescence intensity is approximated to the experimental dependences by variation of a single parameter $k_{0}$. The best fit to the experimental data defines $k_{0}$ as effective absorption coefficient in the central part of the resonance lines. The application of the resonance fluorescence as an analytical tool for atom reactions at low pressures is discussed.
\end{abstract}

Bei reaktionskinetischen Untersuchungen in der Gasphase müssen häufig reaktive Radikale oder Atome bei sehr geringen Teilchendichten qualitativ und quantitativ nachgewiesen werden; dabei soll die Nachweismethode, ohne den Reaktionsablauf zu stören, die einzelnen Reaktionspartner zeitlich unverzögert erfassen. Optische Meßmethoden haben gegenüber anderen Nachweisverfahren, wie z. B. Massenspektrometrie oder Elektronenspin-Resonanz, den Vorteil, daß ohne Störung des Reaktionsablaufes Reaktionszone und Nachweisort identisch sind und hinsichtlich der Gefäßdimensionen keine Beschränkungen auftreten. Besonders bei kleinen Partialdrukken der Reaktionspartner in großen Gefäßen wirken sich optische Methoden vorteilhaft aus, da nicht wie bei den meisten anderen Nachweisverfahren die Konzentration, sondern die optische Schichtdicke = Konzentration $\times$ Weglänge als Meßgröße erfaßt wird.

5ie vorliegende Arbeit beschäftigt sich mit dem optischen Nachweis von reaktiven Sauerstoff- und Stickstoffatomen durch Resonanzfluoreszenz. Bei diesen Atomen liegen die ersten Resonanzniveaus im kurzwelligen Spektralbereich unterhalb von $1800 \AA$. Die Übergangswahrscheinlichkeiten von Atomniveaus im Vakuum-UV-Bereich betragen $10^{7}$ bis $10^{9} \mathrm{~s}^{-1}$. Dementsprechend ergeben sich Absorptionskoeffizienten von $10^{5}$ bis $10^{7}(\mathrm{~cm} \cdot A t m .)^{-1}$, bezogen auf Normalbedingungen. Die hohen Absorptionskoeffizienten erlauben den Nachweis sehr geringer Atomdichten ${ }^{1,2}$. Im Absorptionsspektrum ${ }^{1}$ ist allerdings

1 F. A. Morse u. F. Kaufman, J. Chem. Phys. 42, 1785 [1965].

2 W. Braun u. M. Lenzi, Discussions Faraday Soc. 44, 252 [1967].

3 W. Braun u. T. Carrington, wird veröffentlicht. bei quantitativen Messungen eine relativ große spektrale Auflösung erforderlich, da anderenfalls selbst bei kleinen Konzentrationen kein linearer Zusammenhang zwischen Extinktion und optischer Schichtdicke gegeben ist. Zur optischen Anregung atomarer Resonanzübergänge werden häufig Resonanzlampen verwandt, d. h. Lampen, in denen die gleichen Atome zur Emission ihrer Resonanzlinien in geeigneten Entladungen angeregt werden. Hierbei ist aber die Breite der Emissionslinien infolge von DopplerVerbreiterung im heißen Lampenplasma immer gröBer als die Breite der Absorptionslinie der Atome im Reaktionsgefäß. Unter solchen Bedingungen lassen sich Absorptionsmessungen nur mit Korrekturen durchführen ${ }^{3}$.

Die Schwierigkeit einer starken Doppler-Verbreiterung der Anregungslinie läßt sich umgehen, wenn statt der Absorption die auf sie folgende isotrope Resonanzfluoreszenz gemessen wird. Dabei muß allerdings der Raumwinkel des Detektors so weit eingeengt werden, daß bei Beobachtung der Resonanzfluoreszenz senkrecht zum einfallenden Lichtstrahl kein Streulicht aus der Lampe in den Detektor gelangt.

Sowohl bei Resonanzfluoreszenz- als auch bei Absorptionsmessungen muß mit wachsenden optischen Schichtdicken die Struktur der Resonanzlinien als frequenzabhängige Größe berücksichtigt werden ${ }^{4,5}$.

In der vorliegenden Arbeit wurde untersucht, in welchem Konzentrationsbereich bei gegebenen Gefäßdimensionen die Resonanzfluoreszenz ohne auf-

4 A. C. G. Mitchell u. M. W. Zemansky, Resonance Radiation and Excited Atoms, Cambridge University Press, New York 1934.

5 A. Unsöld, Physik der Sternatmosphären, Springer-Verlag, Berlin 1955. 
wendige Spektralapparate als Nachweismethode anwendbar ist, und wieweit selbst bei nicht aufgelöster Feinstruktur der Resonanzlinien eine lineare $\mathrm{Ab}$ hängigkeit zwischen der Intensität und der Atomkonzentration besteht.

In einem kugelförmigen Reaktionsgefäß wurde die Resonanzfluoreszenz der Edelgase Krypton und Argon und die der Sauerstoff- und Stickstoffatome gemessen. Für die gegebene experimentelle Anordnung wurde ein Modell entwickelt, mit dem die Abhängigkeit der Resonanzfluoreszenzintensität von der Atomkonzentration unter Verwendung der VoigtFunktion ${ }^{4,5}$ berechnet und durch Variation eines Parameters $k_{0}$ an die Meßkurven angepaßt wurde. Die Größe $k_{0}$ hat in diesem Modell die Bedeutung des Absorptionskoeffizienten im Zentrum der Resonanzlinie bei reiner Doppler-Verbreiterung. Die durch $k_{0}$ bestimmten Oszillatorenstärken wurden mit den Werten anderer Messungen - Lebensdauerbestimmungen und Absorptionsuntersuchungen - verglichen.

\section{Experimentelles}

Abbildung 1 zeigt eine Schnittzeichnung der Reaktionskammer. Diese bestand aus einer PyrexglasKugel mit einem Volumen von 12 Litern. Bei den Untersuchungen der Krypton-Resonanzfluoreszenz wurden verschiedene Kryptonmengen in die geschlossene Reaktionskammer eingelassen und mit Argon jeweils auf einen Totaldruck von 2 Torr aufgefüllt. In allen anderen Versuchen strömte das Reaktionsgemisch mit $3 \mathrm{l} / \mathrm{s}$ durch die Kugel. Bei Argon wurden die Drucke durch' Regulierung der Einlaßrate eingestellt. Sauerstoff- oder Stickstoffatome wurden in einem Argon-Sauerstoff- bzw. Argon-Stickstoffgemisch, das durch eine Mikrowellenentladung strömte, erzeugt, wobei der Sauerstoffoder Stickstoffanteil der Mischung höchstens $2 \%$ betrug. Die Atomkonzentrationen bei Sauerstoff und Stickstoff wurden durch Variation des Mischungsverhältnisses verändert. Der Argonzusatz der Mischung wurde auch hier so eingestellt, daß der Totaldruck in der Reaktionskammer 2 Torr betrug. Vor dem Atomeinla $\beta$ in die Kugel war ein zusätzlicher Einlaß angebracht, durch den mit bekannten Zuflußraten mittels einer geeichten Kapillare Stickstoffoxid oder Stickstoffdioxid zur Messung der Partialdrucke von $\mathrm{N}$ - oder O-Atomen dem Reaktionsgemisch zugefügt werden konnte.

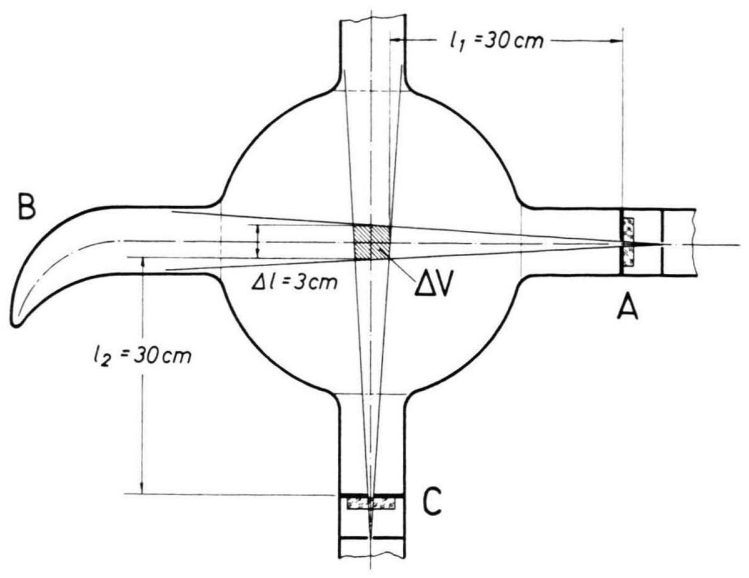

Abb. 1. Schnittzeichnung der Reaktionskammer.

Die Konzentration der N-Atome wurde durch Titration mit NO nach folgendem Reaktionsmechanismus bestimmt ${ }^{6}$ :

$$
\begin{gathered}
\mathrm{N}+\mathrm{NO} \rightarrow \mathrm{N}_{2}+\mathrm{O}, \\
\text { schnelle Titrationsreaktion } \\
\mathrm{NO}+\mathrm{O}+\mathrm{M} \rightarrow \mathrm{NO}_{2}{ }^{*}+\mathrm{M}, \\
\text { Chemilumineszenzreaktion } \\
\text { bei Übertitration mit NO } \\
\mathrm{N}+\mathrm{N}+\mathrm{M} \rightarrow \mathrm{N}_{2}{ }^{*}+\mathrm{M}, \\
\text { Chemilumineszenzreaktion } \\
\text { bei Untertitration mit NO. }
\end{gathered}
$$

Zur Feststellung des Dunkelpunktes im Reaktions. raum diente ein rotempfindlicher Photomultiplier.

Die Sauerstoffatomkonzentration wurde mit $\mathrm{NO}_{2}$ nach folgendem Mechanismus bestimmt ${ }^{6}$ :

$$
\begin{gathered}
\mathrm{O}+\mathrm{NO}_{2} \rightarrow \mathrm{NO}+\mathrm{O}_{2}, \\
\text { schnelle Titrationsreaktion } \\
\mathrm{O}+\mathrm{NO}+\mathrm{M} \rightarrow \mathrm{NO}_{2}{ }^{*}+\mathrm{M}, \\
\text { Chemilumineszenzreaktion, die } \\
\text { überschüssige O-Atome anzeigt. }
\end{gathered}
$$

Am Anschlußstutzen A der Kugel (Abb. 1) befand sich die Resonanzlampe, die ebenfalls mit einer Mikrowellenentladung angeregt wurde. Die Lampenfüllung bestand je nach Versuch aus 1 Torr Krypton, 1 Torr Argon, 1 Torr Argon mit 0,2 Torr Stickstoff oder 1 Torr Argon mit 0,2 Torr Sauerstoff. Ein LiF-Fenster trennte die Lampe vom Reaktionsgefäß. Während des Betriebs wurde die Lampe an einem Kühlfinger mit flüssigem Stickstoff gekühlt. Da die Rekombination von Stickstoffatomen Chemi-

${ }^{6}$ F. Kaufman, Chapter I in G. P. Porter, Progress in Reaction Kinetics, Pergamon Press, London 1961. 
lumineszenz im Vakuum-UV ergibt, war vor dem Lampenfenster ein magnetischer Verschluß eingebaut, so daß mit und ohne Resonanzanregung bei Stickstoffatomen zwischen Resonanzfluoreszenz und Chemilumineszenz unterschieden werden konnte. Das Streulicht der Lampe wurde durch ein Woodsches Horn am Stutzen B der Kugel weitgehend unterdrückt. Eigene aktinometrische Messungen und Literaturwerte ergaben etwa folgende Intensitäten der Resonanzlinien bei einem Lampendurchmesser von 1,5 bis $2,0 \mathrm{~cm}$ mit einem $1 \mathrm{~mm}$ dicken $\mathrm{LiF}$ Fenster, das poliert und bei $400{ }^{\circ} \mathrm{C}$ ausgeheizt war:

Krypton bei $1236 \AA$ : $10^{15}-10^{16}$ Quanten/s ${ }^{7,9,10}$, Argon bei $1067 \AA$ : $10^{13}-10^{14}$ Quanten $/ \mathrm{s}^{8}$, N-Triplett bei $1200 \AA$ : $10^{14}-10^{15}$ Quanten $/ \mathrm{s}^{8,9}$, O-Triplett bei $1305 \AA$ : $10^{14}-10^{15}$ Quanten/s ${ }^{8,10}$.

Krypton emittiert etwa 20\% der Resonanzstrahlung bei $1165 \AA^{7,9,10}$ und Argon etwa 15\% bei $1048 \AA^{8}$.

Senkrecht zur Strahlrichtung der Resonanzlampe war in gleicher Entfernung vom Kugelzentrum am Stutzen C ein Bendix-Multiplier vom Typ M-306 angebracht. Die Wolframkathode dieses Multipliers ist oberhalb von $1500 \AA$ weitgehend lichtunempfindlich. Das Hochvakuumgehäuse des Multipliers war gegen das Reaktionsgefäß mit einem LiF-Fenster abgedichtet. Die Signalimpulse des Detektors wurden gezählt oder zeitlich integriert über einen Gleichspannungsverstärker mit einem Schreiber registriert. Der Raumwinkel von Multiplier und Lampe war auf $8 \cdot 10^{-3}$ sterad eingeengt. Im Reaktionsvolumen $\Delta V$ (Abb. 1) wurden etwa 0,05 bis $0,1 \%$ der Lampenintensität wirksam, wie aktinometrisch mit Hilfe der $\mathrm{N}_{2} \mathrm{O}$-Photolyse ${ }^{11}$ festgestellt wurde. Etwa 2\% des Detektorsignals entstanden durch Streulicht von der Resonanzlampe. Dieser Streulichtanteil gestattete eine Kontrolle der Lampenintensität bei jeder Versuchsserie.

\section{Ergebnisse und Diskussion}

Die Abb. 2-5 zeigen die gemessenen Resonanzfluoreszenzintensitäten in Abhängigkeit von der Konzentration der Atome. Bei Stickstoff wurde die Molekülchemilumineszenz im Vakuum-UV durch Differenzmessung berücksichtigt. Eine Fluoreszenz von metastabilen $\mathrm{N}\left({ }^{2} \mathrm{D}\right)$-Atomen bei 1493 und

11 W. Groth u. H. Schierholz, Planet. Space Sci. 1, 333 [1959]. - G. Greiner, J. Chem. Phys. 47, 4373 [1967].

7 K. H. Becker, Dissertation, Bonn 1964.

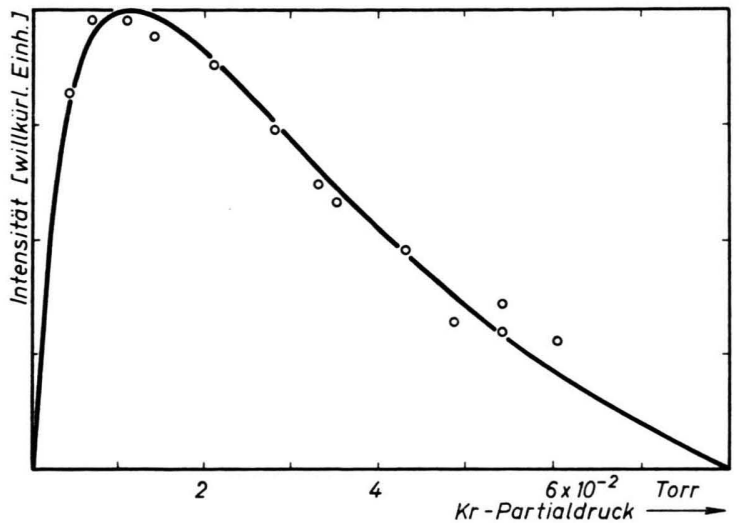

Abb. 2. Abhängigkeit der Kr-Resonanzfluoreszenzintensität von der Atomkonzentration.

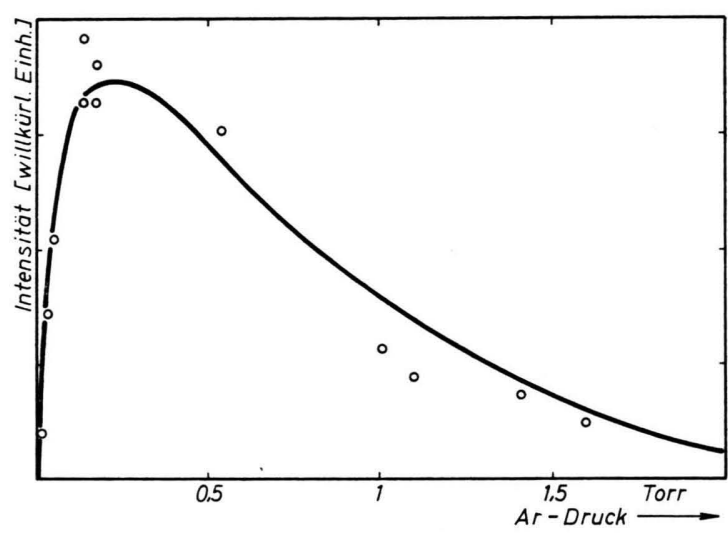

Abb. 3. Abhängigkeit der Ar-Resonanzfluoreszenzintensität von der Atomkonzentration.

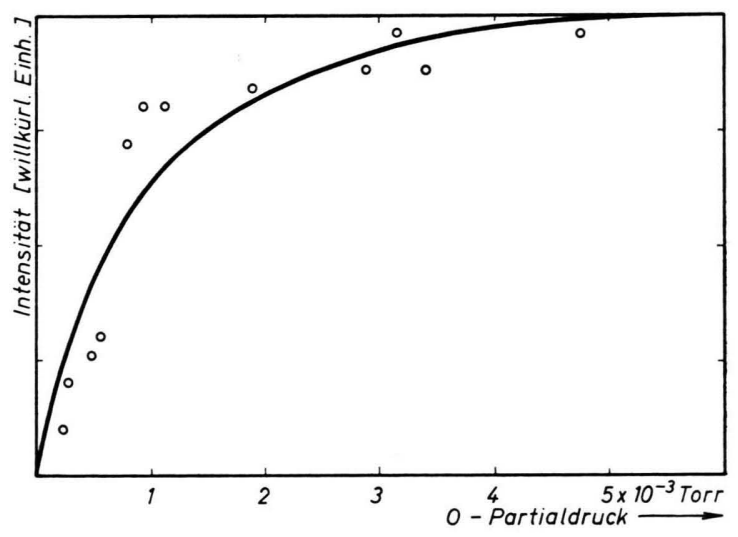

Abb. 4. Abhängigkeit der O-Resonanzfluoreszenzintensität von der Atomkonzentration.

8 M. SCHÜrgers, Diplom-Arbeit, Bonn 1967.

9 H. OKabe, J. Opt. Soc. Am. 54, 478 [1964].

10 D. Davis u. W. Braun, Appl. Opt. 7, 2071 [1968]. 


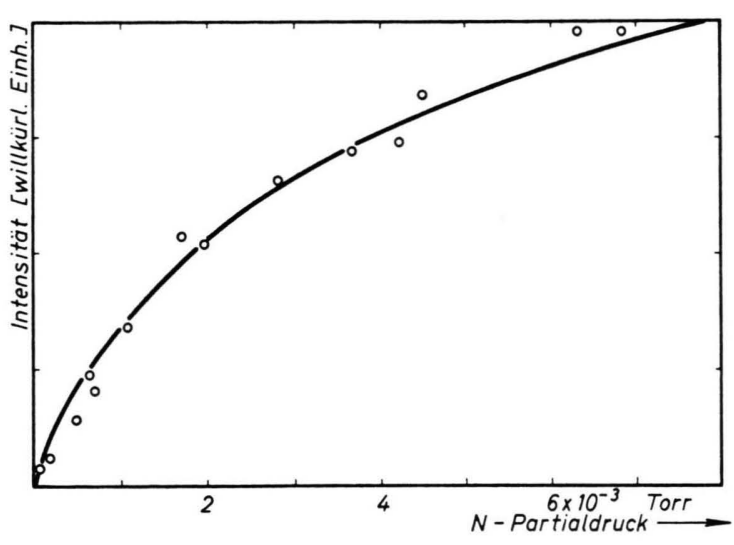

Abb. 5. Abhängigkeit der N-Resonanzfluoreszenzintensität von der Atomkonzentration.

$1495 \AA$ oder von $\mathrm{N}\left({ }^{2} \mathrm{P}\right)$ bei 1743 und $1745 \AA$ konnte ausgeschlossen werden, da ein vor der Anregungslampe verschiebbares $\mathrm{CaF}_{2}$-Fenster, dessen Durchlässigkeitsgrenze bei etwa $1250 \AA$ liegt, die gemessene Intensität bis auf einen Untergrund von $2 \%$ herabsetzte.

Die experimentellen Ergebnisse in Abb. $2-5$ zeigen, daß bis zu folgenden optischen Schichtdicken (Tab. 1) ein lineares Verhalten zwischen Atomkonzentration und Intensität der Resonanzfluoreszenz besteht, wobei als Weglänge der Abstand Kugelzentrum-Lochblende mit $30 \mathrm{~cm}$ eingesetzt wurde:

\begin{tabular}{lc}
\hline \multicolumn{1}{c}{ Atomart } & $\begin{array}{r}\text { optische Schichtdicke } \\
\mathrm{cm} \cdot \text { Atm. }\end{array}$ \\
\hline Krypton & $4 \cdot 10^{-5}$ \\
Argon & $80 \cdot 10^{-5}$ \\
Sauerstoff & $2 \cdot 10^{-5}$ \\
Stickstoff & $9 \cdot 10^{-5}$ \\
\hline
\end{tabular}

Tab. 1.

Eine Abschätzung der maximalen Meßempfindlichkeit ergab für Sauerstoff- und Stickstoffatome, $\mathrm{da} \beta$ bis zu einer optischen Schichtdicke von etwa $5 \cdot 10^{-9} \mathrm{~cm} \cdot$ Atm. unter gleichen experimentellen Bedingungen, d. h. unter Verwendung einer durch Mikrowellenentladung angeregten Resonanzlampe und einem Öffnungswinkel der Lampe und des Empfängers von etwa $8 \cdot 10^{-3}$ sterad, der Nachweis der Resonanzfluoreszenz gerade noch möglich war.

Für die Berechnung der Resonanzfluoreszenzintensität $E_{\Delta v}$, die der photoelektrische Empfänger in willkürlichen, relativen Einheiten anzeigt, wurde ein Modell entwickelt, das als wesentliche Voraussetzung entsprechend den experimentellen Ergebnissen eine lineare Abhängigkeit der Lichtintensität
$E_{\Delta v}$ von der Atomkonzentration bei kleinen Konzentrationen enthält. Die Linienstruktur der Resonanzlinie ist durch den Verlauf des Absorptionskoeffizienten gegeben und unabhängig von der spektralen Verteilung des Anregungslichtes ${ }^{4}$. Das Integral $\int k_{v} \mathrm{~d} v$ über die gesamte Linienbreite der Fluoreszenz stellt für eine gegebene Atomart eine konstante Größe dar, die direkt mit der Übergangswahrscheinlichkeit oder der Oszillatorenstärke zusammenhängt ${ }^{4,5}$, wenn sich $k_{v}$ auf die Konzentrationseinheit, z. B. auf $1 \mathrm{Atm}$. bei $0{ }^{\circ} \mathrm{C}$ bezieht. Die Größe $K_{v}=k_{v} \cdot(\mathrm{N})$ kann dann als konzentrationsabhängig in $\mathrm{cm}^{-1}$ eingeführt werden. Abbildung 1 zeigt unter anderem einen Schnitt der Fläche $\Delta l \cdot \Delta l$ durch das Reaktionsvolumen $\Delta V$ in der Ebene von Lampenstrahl und Beobachtungsrichtung des Detektors. Bei dem relativ kleinen Öffnungswinkel von $8 \cdot 10^{-3}$ sterad können zur Vereinfachung parallele Lichtstrahlen der Anregungslampe im Volumen $\Delta V$ angenommen werden. $\Delta I_{\Delta v}$ sei die im Frequenzbereich $\Delta v$ absorbierte Zahl der Lichtquanten/s im Volumen $\Delta V$. In der Volumeneinheit werden folglich $\Delta I_{\Delta v} / \Delta V$ Atome in der Zeiteinheit elektronisch angeregt. Wegen der kurzen mittleren Lebensdauer $\tau$ bezüglich der Abstrahlung können desaktivierende Stöße vernachlässigt werden. Im Gleichgewicht ergibt sich daher für die Konzentration der angeregten Atome $\left(\mathrm{N}^{*}\right)$ im Volumen $\Delta V$ :

$$
\left(\mathrm{N}^{*}\right)=\tau \cdot \Delta I_{\Delta v} / \Delta V .
$$

Die Intensität der in $\Delta V$ entstehenden Resonanzstrahlung ist proportional zu $\left(\mathrm{N}^{*}\right) \cdot \mathrm{d} V / \tau$, wobei $\mathrm{d} V$ ein Volumenelement aus $\Delta V$ ist. Bei der den Empfänger erreichenden Strahlung muß berücksichtigt werden, daß die Resonanzfluoreszenz sowohl auf dem Weg $\Delta l$ in $\Delta V$ als auch auf dem Weg $l_{2}$ (Abb. 1) absorbiert wird. Bei der Absorption ist der Anteil $K_{v} \mathrm{~d} v /\left(\int_{\Delta v} K_{v} \mathrm{~d} v\right)$ der Resonanzstrahlung im Frequenzbereich $\mathrm{d} v$ in Betracht zu ziehen. Dieser Anteil wird auf dem Weg $l_{2}$ mit $\exp \left(-K_{v} \cdot l_{2}\right)$ geschwächt. Im Volumen $\Delta V$ wird die Absorption durch eine Gewichtsfunktion $\exp \left(-K_{v} \cdot X\right)$ berücksichtigt.

Insgesamt ergibt sich somit für $E_{\Delta v}$ :

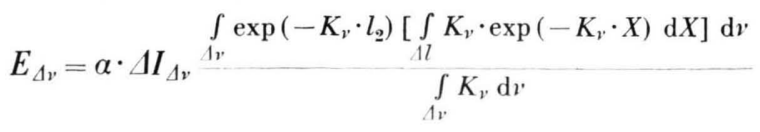

Hierbei bedeuten $l_{2}$ und $\Delta l$ die Absorptionswege in cm und $K_{v}$ ist der Absorptionskoeffizient in $\mathrm{cm}^{-1}$ 
bei einer Atomkonzentration (N). Der Anteil des Resonanzlichtes, das auf dem Wege $l_{2}$ durch Strahlungsdiffusion entsteht, $d . h$. durch mehrfache $\mathrm{Ab}$ sorption und Reemission der vom Volumen $\Delta V$ ausgestrahlten Resonanzfluoreszenz, wird in diesem Modell nicht berücksichtigt. Wie das Ergebnis zeigt, scheint bei kleinem Öffnungswinkel und relativ niedrigen Atomkonzentrationen diese Vernachlässigung berechtigt zu sein. Für $\Delta I_{\Delta v}$ wurden verschiedene Ansätze durchgerechnet. Die Annahme, daß die Anregungsquelle eine Linie mit sehr starker Selbstumkehr ausstrahlt, führt zu der besten Übereinstimmung mit der experimentell gefundenen Abhängigkeit. In diesem Fall wird das Anregungslicht nur schwach im Reaktionsgefäß absorbiert und der in $\Delta V$ absorbierte Teil kann mit $\gamma \cdot(\mathrm{N}) \cdot \Delta l$ angesetzt werden; bis auf den Partialdruck (N) sind dieses für eine Atomart konstante Größen bei gegebener Lampenintensität. Mit diesem Ansatz ergibt sich für $E_{\Delta v}$ :

$$
\begin{aligned}
& E_{\Delta v}=\mathrm{C} \int_{\Delta v} \exp \left(-k_{v} \cdot(\mathrm{N}) \cdot l_{2}\right) \\
& \cdot\left[1-\exp \left(-k_{v} \cdot(\mathrm{N}) \cdot \Delta l\right)\right] \mathrm{d} v .
\end{aligned}
$$

Die Größe $C$ enthält alle Konstanten wie Geometriefaktoren, Intensität der Anregungslampe und Übergangswahrscheinlichkeit des Resonanzüberganges.

Die Struktur von $k_{v}$ wird durch Doppler-Verbreiterung $\left(\Delta v_{\mathrm{D}}\right)$, durch Stoßverbreiterung $\left(\Delta v_{\mathrm{S}}\right)$ und durch die natürliche Linienbreite $\left(\Delta v_{\mathrm{N}}\right)$ bestimmt ${ }^{4,5}$.

Mit Hilfe der sogenannten Voigt-Funktion kann das Integral (1) ausgewertet werden.

$$
\begin{aligned}
k_{\nu} & =k_{0}(a / \pi) \cdot \int_{-\infty}^{+\infty} \exp \left(-y^{2}\right)\left[a^{2}+(\omega-y)^{2}\right]^{-1} \mathrm{~d} y \\
a & =\left[\left(\Delta v_{\mathrm{N}}+\Delta v_{\mathrm{S}}\right) / \Delta v_{\mathrm{D}}\right] \cdot(\ln 2)^{1 / 2} \\
\omega & =2\left[\left(v_{0}-v\right) / \Delta v_{\mathrm{D}}\right] \cdot(\ln 2)^{1 / 2} \\
\Delta v_{\mathrm{D}} & =2,15 \cdot 10^{4} \cdot\left(\lambda_{0}\right)^{-1} \cdot(T / M)^{1 / 2}
\end{aligned}
$$

$\lambda_{0}$ ist hierbei die Wellenlänge im Linienzentrum in cm, $M$ das Atomgewicht und $T$ die Temperatur in ${ }^{\circ}$ K. $k_{0}$ in Gl. (2) stellt den Absorptionskoeffizienten im Zentrum der Resonanzlinie bei reiner DopplerStruktur dar, bezogen auf Normalbedingungen.

Nach der Methode der kleinsten quadratischen Abweichung wurde das Integral (1) unter Variation des Normierungsfaktors $C$, des Dämpfungsverhältnisses $a$ und des Parameters $k_{0}$ an die experimentell ermittelte Abhängigkeit angepaßt. In Übereinstimmung mit älteren Arbeiten über Fluoreszenzuntersuchungen ${ }^{4}$ ergab sich, daß das Dämp- fungsverhältnis $a$ in der Größenordnung von $10^{-2}$ lag. Es zeigte sich, daß bei den relativ geringen Stickstoff- und Sauerstoffkonzentrationen eine Veränderung von $a$ im Bereich von $10^{-2}$ keinen wesentlichen Einfluß auf den ermittelten Kurvenverlauf ergab. Für Krypton und Argon wurde mit $a=10^{-2}$ eine ausreichende Näherung an die experimentellen Abhängigkeiten erzielt.

In Abb. 2-5 sind die berechneten Kurven (ausgezogene Linie) und die einzelnen Meßpunkte dargestellt. Die gute Übereinstimmung mit den Meßwerten zeigt, daß das entwickelte Modell die Druckabhängigkeit der Resonanzstrahlung unter den vorliegenden Bedingungen befriedigend beschreibt. Demnach sollten die als Parameter über Gl. (2) eingeführten $k_{0}$-Werte Oszillatorenstärken $f$ bzw. Summen über die Oszillatorenstärken multipliziert mit den statistischen Gewichten $\Sigma g_{n} \cdot f_{n m}{ }^{4,5}$ aller beteiligten Resonanzübergänge ergeben, die größenordnungsmäßig mit den Werten aus Absorptionsmessungen und direkten Lebensdauerbestimmungen übereinstimmen.

Die Oszillatorenstärken lassen sich nach Gl. (3) berechnen:

$$
\begin{aligned}
& \int_{\Delta v} k_{v} \mathrm{~d} v=2,5 \cdot 10^{-2} \cdot \Sigma f_{n m} \cdot\left(\mathrm{N}_{n}\right), \\
& \int_{\Delta v} k_{\nu} \mathrm{d} v=\Delta v_{\mathrm{D}} \cdot \sum k_{0}{ }^{n m} \cdot\left(\mathrm{N}_{n}\right) /\left(\mathrm{N}_{0}\right) ;
\end{aligned}
$$

$\left(\mathrm{N}_{n}\right)$ ist die Besetzungsdichte eines absorbierenden Zustandes mit $\Sigma\left(\mathrm{N}_{n}\right)=\left(\mathrm{N}_{0}\right)$, wobei $\left(\mathrm{N}_{0}\right)$ die Zahl der Atome $/ \mathrm{cm}^{3}$ bei $1 \mathrm{Atm}$. und $0{ }^{\circ} \mathrm{C}$ ist.

Tabelle 2 zeigt, daß dies für die Resonanzübergänge der Edelgasatome recht gut erfüllt ist. Sowohl bei Stickstoff $N\left({ }^{4} \mathrm{P}_{1 / 2,3 / 2,5 / 2} \longleftrightarrow{ }^{4} \mathrm{~S}_{3 / 2}\right)$ als auch bei Sauerstoff $\mathrm{O}\left({ }^{3} \mathrm{~S}_{1} \longleftrightarrow{ }^{3} \mathrm{P}_{0,1,2}\right)$ besitzen die Resonanzlinien eine Triplett-Feinstruktur. Für Stickstoff sind die $f_{n m}$ entsprechend den statistischen Gewichten der drei oberen Zustände verschieden. Bei der Summation über das Triplett kann $\Sigma f_{n m} \cdot\left(\mathrm{N}_{n}\right)=$ $\left(\mathrm{N}_{0}\right) \cdot \Sigma f_{n m}$ näherungsweise nach Gl. (3) auf $\int k_{\nu} \mathrm{d} v=3 k_{0} \Delta \nu_{\mathrm{D}}$ bezogen werden, wobei $k_{0}$ einen mittleren Absorptionskoeffizienten für alle drei Linien darstellt. Bei Sauerstoff ist der Grundzustand $0\left({ }^{3} \mathrm{P}\right)$ durch drei Niveaus $J=0,1,2$ gekennzeichnet. Da der obere Zustand $\left({ }^{3} \mathrm{~S}\right)$ nicht aufgespalten ist, sind die $f_{n m}$ aller drei Triplett-Linien gleich, die Besetzungsdichten $\left(\mathrm{N}_{n}\right)$ der Niveaus des Grundzustandes verschieden. Demnach führt die Summation über das gesamte Triplett zu $\Sigma f_{n m} \cdot\left(\mathrm{N}_{n}\right)=f \cdot\left(\mathrm{N}_{0}\right)$ mit $\int_{\Delta v} k_{v} \mathrm{~d} v=k_{0} \cdot \Delta v_{\mathrm{D}}$. Trotz Triplettaufspaltung ist 


\begin{tabular}{|c|c|c|c|}
\hline Atomart & $\begin{array}{c}k_{0} \\
(\mathrm{~cm} \cdot \mathrm{Atm} .)^{-1}\end{array}$ & $\begin{array}{c}f \text { bzw. } \\
\Sigma g_{n} \cdot f_{n m}\end{array}$ & Literatur \\
\hline $\begin{array}{l}\text { Krypton } \\
\quad[1236 \AA]\end{array}$ & $\begin{array}{c}- \\
- \\
- \\
\overline{-} \\
4,1 \cdot 10^{7}\end{array}$ & $\begin{array}{l}f \\
0,158 \\
0,266 \\
0,152 \\
0,166 \\
0,2\end{array}$ & $\begin{array}{c}12 \\
13 \\
14 \\
15 \\
\text { diese Arbeit }\end{array}$ \\
\hline $\begin{array}{l}\text { Argon } \\
\quad[1067 \AA]\end{array}$ & $\begin{array}{c}- \\
2,1 \cdot 10^{6}\end{array}$ & $\begin{array}{l}f \\
0,05 \\
0,026 \\
0,017\end{array}$ & $\begin{array}{c}16 \\
17 \\
\text { diese Arbeit }\end{array}$ \\
\hline $\begin{array}{l}\text { Sauerstoff } \\
{\left[\begin{array}{ll}1302 & \AA \\
1305 & \AA \\
1306 & \AA\end{array}\right]}\end{array}$ & $\begin{array}{c}- \\
- \\
- \\
-\overline{8} \cdot 10^{7}\end{array}$ & $\begin{array}{l}\sum g_{n} \cdot f_{n m} \\
0,3 \\
0,3 \\
0,425 \\
1,62 \\
7,6\end{array}$ & $\begin{array}{c}1 \\
18 \\
19 \\
20 \\
\text { diese Arbeit }\end{array}$ \\
\hline $\begin{array}{l}\text { Stickstoff } \\
{\left[\begin{array}{ll}1199,6 & \AA \\
1200,2 & \AA \\
1200,7 & \AA\end{array}\right]}\end{array}$ & $\begin{array}{c}\overline{-} \\
- \\
\overline{-} \\
1.7 \cdot 10^{7}\end{array}$ & $\begin{array}{l}\sum g_{n} \cdot f_{n m} \\
0,39 \\
0,2 \\
1,04 \\
1,408 \\
2,5\end{array}$ & $\begin{array}{c}18 \\
1 \\
21 \\
22 \\
\text { diese Arbeit }\end{array}$ \\
\hline
\end{tabular}

Tab. 2.

die Übereinstimmung für Stickstoffatome, vor allem mit dem Wert von LABUHN ${ }^{22}$, noch befriedigend; dagegen liegen die Oszillatorenstärke bzw. $\Sigma g_{n} \cdot f_{n m}$, die sich aus dem $k_{0}$ dieser Arbeit für Sauerstoffatome ergeben, wenigstens fünffach höher als die entsprechenden Werte anderer Autoren. Andererseits wird die Konzentrationsabhängigkeit für Sauer-

12 P. G. Wilkinson, J. Quant. Spectr. Radiative Transfer 5, 503 [1965].

13 J. КосH, Kgl. Fysiograf. Sällskap Lund, Förh. 19, 173 [1949].

14 J. D. Dow u. R. S. KNox, Phys. Rev. 152, 152 [1966].

15 R. Turner, Phys. Rev. 140, A 426 [1965].

16 A. Gold u. R. S. Knox, Phys. Rev. 113, 834 [1959].

17 J. L. Morack u. C. E. Fairchild, Phys. Rev. 163, 125 [1967]. stoff durch das Integral (1) mit $k_{0}=8 \cdot 10^{7}$ (cm.Atm. $)^{-1}$ recht gut beschrieben, wie Abb. 4 zeigt. Ein Vergleich der gemessenen Abhängigkeiten für die verschiedenen Atomarten (Abb. 2-5) läßt deutlich erkennen, daß der effektive $k_{0}$-Wert für das Sauerstoff-Triplett größer als die Werte für die übrigen Resonanzübergänge sein muß. Vermutlich ist die Summation über die Triplettstruktur, vor allem bei Sauerstoffatomen, für die $f$-Bestimmung eine unzureichende Näherung.

Mit entsprechender spektraler Triplettauflösung könnten die $k_{0}$ - bzw. die $f$-Werte bei Untersuchungen der Resonanzfluoreszenz sicherlich mit größerer Genauigkeit bestimmt werden. Die vorliegende Arbeit sollte vor allem die Anwendungsmöglichkeiten der Resonanzfluoreszenz für analytische Zwecke aufzeigen. Demnach kann besonders bei niedrigen Atomkonzentrationen die Resonanzfluoreszenz ohne allzu großen experimentellen Aufwand als Nachweismethode eingesetzt werden; für Absolutbestimmungen der Atomkonzentration ist diese Methode allerdings weniger geeignet.

Herrn Dipl.-Math. HundT sei für seine Hilfe bei der Durchführung des Rechenprogramms mit einer IBMGroßrechenanlage vielmals gedankt. - Diese Arbeit wurde durch finanzielle Unterstützung des Bundesministeriums für wissenschaftliche Forschung und des Landesamtes für Forschung des Landes Nordrhein-Westfalen ermöglicht.

18 A. B. Prag, C. E. Fairchild u. K. C. Clark, Phys. Rev. 137, A 1358 [1965].

19 G. M. Lawrence, Bull. Am. Phys. Soc. 13, 424 [1968].

20 G. Boldt u. F. Labuhn, Z. Naturforsch. 22 a, 1613 [1967].

21 G. M. Lawrence u. B. D. Savage, Phys. Rev. 141, 67 [1966].

22 F. LabUhn, Z. Naturforsch. 20 a, 998 [1965]. 